\title{
ISOMERIZATION OF TRANS-DIETHYLSTILBESTROL TO PSEUDO-DIETHYLSTILBESTROL
}

Subhash C. Airy and Joseph E. Sinsheimer

College of Pharmacy, University of Michigan, Ann Arbor, Michigan 48109

Received 9-28-81

\section{ABSTRACT}

This study reports the formation and isolation of a diethylstilbestrol-dimethylsulfoxide (DES-DMSO) adjunct and Z-3,4di(p-hydroxyphenyl)-2-hexene ( $\psi$-DES) from trans-DES. The presence of $\psi$-DES was indicated by NMR and mass spectrometry and confirmed by direct comparison to a reference sample. High resolution $\operatorname{NMR}(360 \mathrm{MHz})$ along with the comparison of the chemical shift values of methine and methyl protons attached to carbon-carbon double bond $s$ in $Z$ and $E$ isomers of 3-substituted-2-pentenes and dienestrol derivatives were used in postulating the $Z$-stereocheristry for $\psi$-DES. A NMR additive increment method was useful for the comparison of the chemical shift values of methine protons in $\psi$-DES and other literature compounds. Nuclear Overhauser Enhancement (NOE) confirmed the $Z$-stereochemistry of $\psi$-DES.

\section{INTRODUCTION}

Metabolic activation is an important consideration in chemical toxicity. Many ethylenic compounds result in the formation of reactive epoxides which have been assumed to be possible active metabolites. Since DES is a human carcinogen, it was of interest to include its trans and cis epoxide derivatives in our mutagenicity study of aliphatic epoxides reported earlier (1). Therefore, the trans to cis isomerization of DES (2) was reexplored under various reaction conditions. As part of this 
investigation, a DES-DMSO adjunct and $\psi$-DES were isolated

from trans-DES under mild conditions.

EXPERIMENTAL

Apparatus

Melting points were measured on a Mel-Temp melting point apparatus and wers uncorrected. IR spectra were recorded on Perkin Elmer 281 spectrophotometer in KBr pellets. NMR spectra were obtained with a Varian EM -360 at $60 \mathrm{MHz}$ using tetramethylsilane as an internal standard. High resolution proton NMR spectra were recorded with Bruker $W M-360$ at $360 \mathrm{MHz}$. For NOE experiments, the dissolved oxygen from air was thoroughly removed from the specimen in the NMR tube. This was accomplished by first freezing the specimen in liquid nitrogen followed with careful thawing under vacuum. The above step was repeated eight times until air bubbles ceased coming out of the frozen specimen. The NMR tube was then carefully sealed. The solid state carbon-13 NMR spectra were obtained by Dr. Warner R. Woolfenden (4). Differential scanning calorimetry (DSC) were recorded from a Perkin Elmer DSC-2C instrument with the following settings: heating rate $20^{\circ} \mathrm{K} / \mathrm{min}$.; range setting $10 \mathrm{mcal} / \mathrm{sec}$.; Indium standard was used to calibrate the calorimeter. Thermogravimetric analysis (TGA) data were recorded with Perkin Elmer TGS-2 instrument with the following settings:heating rate $5^{\circ} \mathrm{C} / \mathrm{min}$.; range setting 0.1. Mass spectra(70eV) were obtained with Finigan $4023 \mathrm{GC}$ MS with INCOS data acquisition system. UV spectrum was measured in methanol with Hewlett Packard 8450 A spectrophotometer.

$z-3,4-\operatorname{di}(p-h y d r o x y p h e n y l)-2$-hexene $(\psi-$ DES $)$

Trans-DES (ICN Pharmaceutical, Inc.) (3.0g) was added to dimethylsulfoxide $(25 \mathrm{ml})$ and the mixture was heated on a steam bath for three minutes. The contents were diluted with distilled water $(50 \mathrm{ml})$ and filtered immediately. The filtrate was then left for over three weeks where fine crystals of $\psi-D E S$ was isolated and dried under vacuum. Yield $178 \mathrm{mg}(5.9 \%) ; \mathrm{m}$. p. $151-152^{\circ} \mathrm{C}$ (1it., $153^{\circ} \mathrm{C}$ and $143.5^{\circ} \mathrm{C}(5)$ reported for unspecified stereo-isomers of $\psi$-DES); IR (KBr) 3280 (broad), 2960, $2920,2860,1870$ (weak, shoulder), 1610, 1595, 1500, 1440,1365 (with shoulder), 1230 (broad), $1170,1120,1010,940,925,815 \mathrm{~cm}^{-1}$. 
$360 \mathrm{MHz}$ NMR $\left(\mathrm{d}_{6}\right.$-acetone); $\delta 0.85\left(\mathrm{t}, \mathrm{J}=7.3 \mathrm{~Hz}, 6-\mathrm{CH}_{3}\right)$, $1.48\left(\mathrm{~d}, \mathrm{~d}, \mathrm{~J}=6.7 \mathrm{~Hz}, \mathrm{~J}=0.9 \mathrm{~Hz}, 1-\mathrm{CH}_{3}\right), 1.65(\mathrm{~m}, 5-\mathrm{H}), 1.77$ $(\mathrm{m}, 5-\mathrm{H}), 3.3 \mathrm{I}(\mathrm{t}, \mathrm{J}=7.3 \mathrm{~Hz}, 4-\mathrm{H}), 5.57(\mathrm{q}, \mathrm{q}, J=6.7 \mathrm{~Hz}, J=0.9$ $\mathrm{Hz}, 2-\mathrm{H}), 6.70(\mathrm{~m}$, aromatic, $6 \mathrm{H}), 6.93(\mathrm{~m}$, aromatic, $2 \mathrm{H})$, $8.07(\mathrm{~s}$, phenolic $\mathrm{OH}), 8.18(\mathrm{~s}$, phenolic $\mathrm{OH})$. Double irradiation at $\delta 3.31 \mathrm{ppm}$; observed at $\delta 1.48\left(\mathrm{~d}, 1-\mathrm{CH}_{3}\right)$ and $\delta 5.57(\mathrm{q}, 2-\mathrm{H})$. Mass spectrum $\mathrm{m} / \mathrm{e}$ (relative intensity) $268\left(\mathrm{M}^{+}, 32\right), 239(80)$, $145(57), 135(35), 134(12), 133(79), 121(13), 107(100), 105$ $(18), 91(18), 77(30), 55(15)$.

\section{Diethylstilbestrol-Dimethylsulfoxide Adjunct (DES-DMSO)}

The initial residue from the above experiment was thoroughly washed with carbon tetrachloride until the washings did not show the presence of DMSO as checked by proton NMR. The residue was dried under vaculum $(0.5 \mathrm{~mm}$ of $\mathrm{Hg})$ at $110^{\circ} \mathrm{C}$ for 18 hours. Yield $3.6 \mathrm{~g}(93 \%) ; \mathrm{m} . \mathrm{p} .144-145^{\circ} \mathrm{C} ; 60 \mathrm{MHz}$ NMR (d 6 -acetone); $\delta 0.75$ (t, J $\left.=7.0 \mathrm{~Hz}, \mathrm{CH}_{3}\right), 2.17\left(\mathrm{q}, \mathrm{J}=7.0 \mathrm{~Hz}, \mathrm{CH}_{2}\right), 2.60$ (s, $\mathrm{CH}_{3}$ of DMSO), $6.90(\mathrm{~m}$, aromatic, $8 \mathrm{H}), 8.43(\mathrm{~s}$, phenolic OH); carbon - 13 NMR (acetone); 13.7, 29.1, 40.8 (DMSO), 115.6, 130.4, 134. 3, 139.4, 156.6; solid state carbon-13 MAS/CP NMR (4); $14.5,27.3,37.6,115.3,116.6,129.8,133.2,135.1,138.2$, $155.2 \mathrm{ppm}$. IR (KBr); 3410, 3270, 2980, 2940, 2875, 1992, 1610 , $1591,1515,1455,1440,1430,1370,1355,1338,1282.1248$, $1235,1205,1175,1115,1010,956,850,830,800,745,732$, $718,640 \mathrm{~cm}^{-1}$. Mass spectrum m/e(relative intensity); $268\left(\mathrm{M}^{+}\right.$, $52), 239(36), 159(22), 145(76), 133(21), 121(29), 115$ (19), $107(100), 91(20), 77(26)$.

Anal. (Midwest Microlab, Indianapolis, IN) Calcd for $\mathrm{C}_{20} \mathrm{H}_{26} \mathrm{O}_{3} \mathrm{~S}: \mathrm{C}, 69.36 ; \mathrm{H}, 7.51 ; \mathrm{S}, 9.24 \%$. Found: C, $69.77, \mathrm{H}, 6.88, \mathrm{~S}, 9.47 \%$.

\section{RESULTS AND DISCUSSION}

In our modification of the literature procedure of the isomerization in DMSO of trans-DES to cis-DES (2), a DES-DMSO adjunct can be isolated immediately upon addition of water to the reaction mixture. This is followed after several weeks by precipitation of $\psi$-DES from the filtrate. Even under conditions 
of washing and drying to remove any free DMSO, the initial precipitate showed the presence of sulfur by elemental analysis. Mass spectra of the precipitate and trans-DES were the same. Solution spectra were consistent with either a mixture of DMSO and trans-DES or an adjunct of the two which was unstable in solution. That is, UV analysis in methanol gave a spectrum consistent with that of trans-DES and NMR in deuterated methanol or acetone gave spectra indicating a $1: 1$ ratio of DMSO to trans-DES. An IR spactrum obtained from a solid state sample in $\mathrm{KBr}$ confirmed the presence of DMSO with several bands not present in a trans-DES spectrum. A second $O H$ stretching band at $3270 \mathrm{~cm}^{-1}$ and a new band at $1235 \mathrm{~cm}^{-1}$ (hydrogen bonded $\mathrm{OH}$ bending (3)) as well as a strong increase in absorbance at $1010 \mathrm{~cm}^{-1}$ (bonded $\mathrm{S}=0$ stretching (3)) all suggest a DESDMSO adjunct. Also the precipitate in question behaved like a single compound exhibiting a sigmoidal curve in TGA without any weight loss until $116.5^{\circ} \mathrm{C}$ while DMSO under the same conditions exhibited $99.5 \%$ loss of its total weight at $116^{\circ} \mathrm{C}$. DSC analysis for the precipitate showed a sharp endotherm characteristic for a pure compound at $147^{\circ} \mathrm{C}$ as compared to $173^{\circ} \mathrm{C}$ for the trans-DES.

Solid state carbon-13 magic angle spinning/cross polarization (MAS/CP) nuclear magnetic resonance spectra for the 
DES-DMSO adjunct as well as for trans-DES were obtained (4) and are summarized in Table 1. The DES-DMSO adjunct exhibited the greatest difference in chemical shift value $(\Delta \delta=2,70 \mathrm{ppm})$ for the carbon attached to a phenolic group. This is consistent with hydrogen bonding of the phenolic protons to the oxygen of DMSO. The spectrum of the DES-DMSO adjunct also confirmed a 1:1 ratio of DMSO to DES.

Initial IR, NMR and mass spectra of the second precipitate led us to the conclusion that we were dealing with $\psi$-DES. This was confirmed by comparision of our $60 \mathrm{MHz}$ NMR spectrum to the one furnished by K.S. Korach for the $\psi$-DES used in his study (6) as well as by direct comparison of $360 \mathrm{MHz} N M R$ and mass spectra of our compound to that of a reference sample (7) of $\psi$-DES.

The mass spectrum of our isolated $\psi$-DES indicated important peaks not included in an abridged spectrum previously reported (8). We rationalize the fragmentation pattern as outlined in Scheme 1. Various investigators $(5,8-12)$ have reported the preparation of $\psi$-DES but without evidence for its $E$ or $Z$ stereochemistry. In the present study the stereochemistry of the isolated and reference $\psi$-DES was predicted on the basis of comparison to literature NMR data for mono-mcthyl-trisubstituted alkencs 

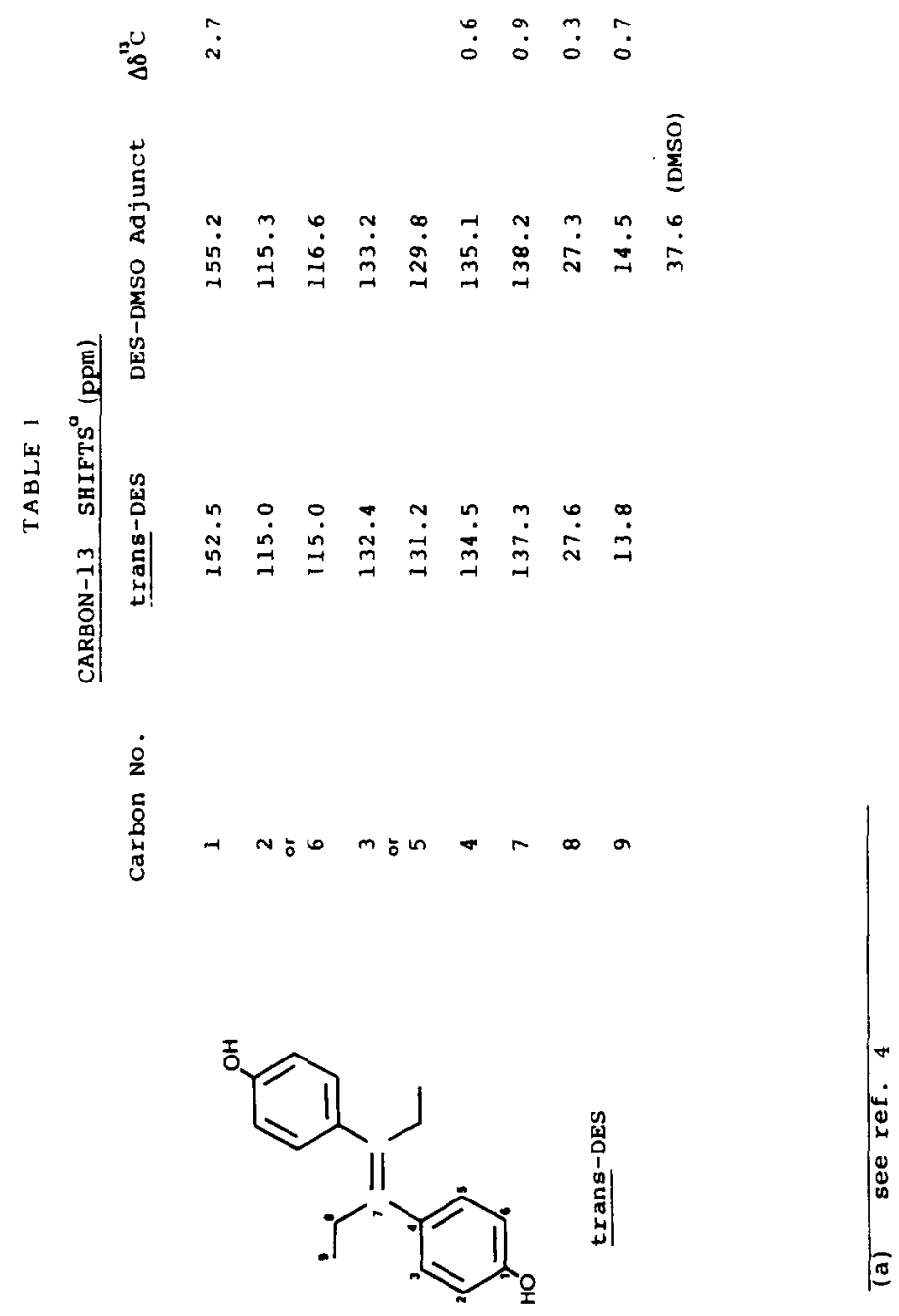


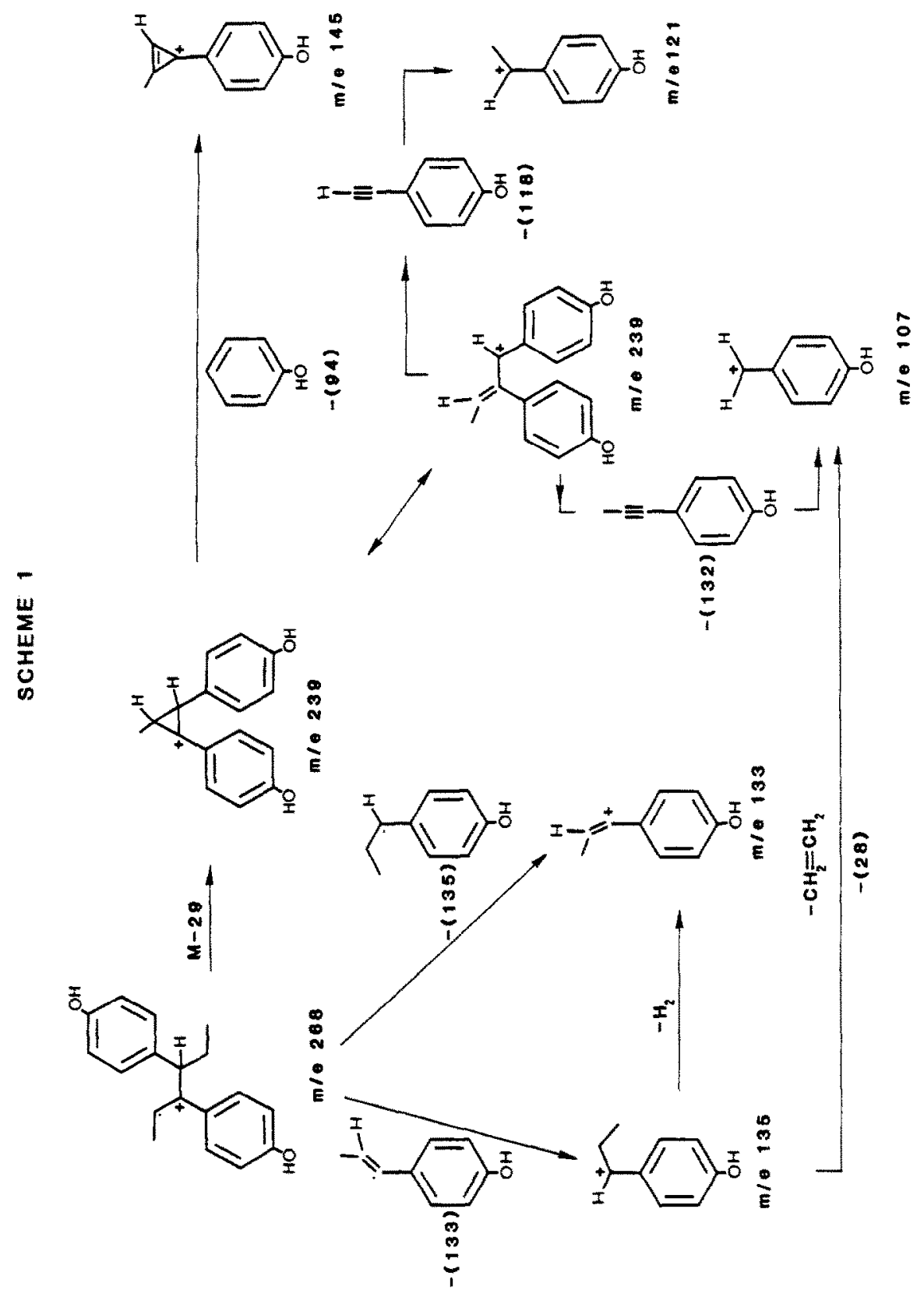


and confirmed by NOE measurements to be the $\mathrm{Z}$-isomer.

We applied the additive increment method of Pascual

et al. (13) and Matter et al. $(14,15)$ to $\psi$-DES and predicted the chemical shift for the methine proton to be $\delta 5.77 \mathrm{ppm}$ for the $E$-isomer and $\delta 5.41 \mathrm{ppm}$ for the $Z$-isomer. As is generally the case in the observed $(16,17)$ and our calculations for the methine protons in the configuration about the double bonds of this series of trisubstituted alkenes, the predicted value for the $\mathrm{Z}$-isomer was less than that of the E-isomer. Thus, our observed value of $\delta 5.57 \mathrm{ppm}$ best fits the calculated value of $\delta 5.41 \mathrm{ppm}$ for the $Z$-isomer of $\psi$-DES.

Also our observed value of $\delta 1.48 \mathrm{ppm}$ for the methyl group attached to the double bond of $\psi$-DES corrosponds more closely to the observed value of $\delta 1.45$ to $\delta 1.56$ for these protons of the $\mathrm{Z}$-isomers in the same series of trisubstituted alkenes (16) than values of $\delta 1.78$ to $\delta 1.88$ for $E$-isomers. This $Z$ assignment is further supported by methyl proton values of $\delta 1.45 \mathrm{vs} \delta 1.70$ for conformation about each double bond in dienestrol diacetate in a study in which Metzler (17) noted the differences in chemical shifts of both the methyl and methine protons in a series of dienestrol derivatives.

The presence of a single asymmetric center at the $C-4$ position (Fig. 1) is clearly shown by the non-equivalence display 


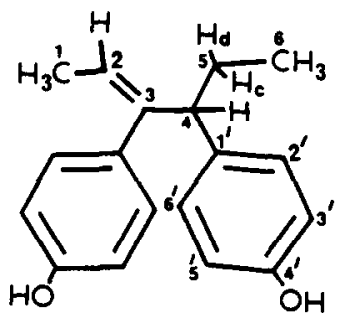

Figure 1

of the protons of the adjacent methylene group. Thus a noteworthy feature of the NMR spectrum of $\psi$-DES is that each methylene protons exhibited different chemical shift ( 81.77 and 81.65$)$ with multiple spiitting.

In $\Psi$-DES long range proton-proton coupling constants were also detected. Our double irradiation experimen:s show that the olefinic hydrogen atom and methyl protons attached to the carboncarbon double bond are coupled with the methine proton at C -4 (Fig. 1). The above protons are split by the methine proton with an equal coupling constant $(\mathrm{J}=0.9 \mathrm{~Hz})$. However, after decoupling at $63.3 \mathrm{ppm}$ (methine proton), the methyl as well as olefinic protons exhibited a sharp doublet and a quartet respectively.

NOE measurements (Table 2) were extremely useful in the confirmation of the stereochemistry of the olefinic side chain in $\Psi$-DES. The $Z$-stereochemistry is supported by comparing the 
TABLE 2

NUCLEAR OVERHAUSER ENHANCEMENTS IN $\Psi$-DES

\begin{tabular}{|c|c|c|}
\hline Irradiate & Observe & NOE, \% \\
\hline $\mathrm{H}-2$ & $\mathrm{H}-5 \mathrm{~d}$ & 40.9 \\
$\mathrm{H}-2$ & $\mathrm{H}-5 \mathrm{c}$ & 21.7 \\
$\mathrm{CH}_{3}-1$ & $\mathrm{H}-5 \mathrm{~d}$ & 27.3 \\
$\mathrm{CH}_{3}-1$ & $\mathrm{H}-5 \mathrm{c}$ & 17.4 \\
$\mathrm{H}-4$ & $\left(\mathrm{H}-2^{\prime}+\mathrm{H}-6^{\prime}\right)$ & 5.0 \\
\hline
\end{tabular}

magnitude of the NOEs for $\left(\mathrm{CH}_{3}-1\right), \mathrm{H}-5 \mathrm{~d}=27.3 \%$ (read as saturation of $\mathrm{CH}_{3}-1$ protons caused a $27.3 \%$ increase of the signal from proton $\mathrm{H}-5 \mathrm{~d}),\left(\mathrm{CH}_{3}-1\right), \mathrm{H}-5 \mathrm{c}=17.4 \%$ and $(\mathrm{H}-2)$, $\mathrm{H}-5 \mathrm{~d}=40.9 \%,(\mathrm{H}-2), \mathrm{H}-5 \mathrm{c}=21.7 \%$. These NOE data clearly imply that $\mathrm{CH}_{3}-1$ group is cis oriented in relation to the phenyl group and the olefinic proton $(\mathrm{H}-2)$ is more proximate to the methylene protons (Fig. 1). Additional virtues of the NOE data are shown in the spectral assignments that could be made for the aromatic protons at $66.93(2 \mathrm{H})$ which is shown by the observance of the $\operatorname{NOE}(H I-4), H I-2^{\prime}+I I-6^{\prime}=5 \%$.

\section{ACKNOWLEDG MENTS}

This study was supported in part by Grant number 1 R0 1 CA 25770, awarded by the National Cancer Institute, DHHS. The authors wish to express their appreciation to $\mathrm{Dr}$. Warner $\mathrm{R}$. Woolfenden, the University of Utah for supplying the solid state carbon-13 MAS/CP NMR spectra and Dr.M.G. Ganesan, the University of Michigan for the TGA and DSC data. 


\section{REFERENCES}

1. Wade, D.R., Airy, S.C. and Sinsheimer, J.E., MUTATION RES., $58,217(1978)$.

2. White, W.A. and Ludwig, N.H., J.AGRI. FOOD CHEM., 19, 388(1971).

3. Bellamy, L.J.,THE INFRARED SPECTRA OF COMPLEX MOLECULES, Chapman and Hall, London (1975) p. 124, 402.

4. Personal communication from $\mathrm{Dr}$. Warner $\mathrm{R}$. Woolfenden, Dept. of Chemistry, The University of Utah, Salt Lake City, Utah 84112 .

5. Wessely, F., Kerschbaum, E., Kleedorfer, A., Prillinger, F. and Zajic, E., MONATSCH, 73,127 (1940).

6. Korach, K.S., Metzler, M. and Mclachlan, J.A., J.BIOL. CHEM., 254,8963 (1979).

7. Reference sample was provided through the courtesy of R. Villotti and F.Fontani, Recordati, Milano, Italy.

8. Tennent, D.M., Kouba, R.F., Ray, W.H., Vanden Heuvel, W.J.A. and Wolf, F.J., SCIENCE, 194, 1059 (1976).

9. Walton, E. and Brownlee, G., NATU $\overline{R E}, 151,305$ (1943).

10. Dodds, E.C., Goldberg, L., Lawson, W. and Robinson, R., PROC. ROYAL SOC. LONDON, B127, 140 (1939).

11. Solmssen, U.V., CHEM. REV., 481 (1945).

12. Gottschlich, R. and Metzler, M., ANAL. BIOCHEM., 92 , $199(1979)$.

13. Pascual, C., Meir, J. and Simon, W., HELVETICA CHIMICA ACTA, 49, 164 (1966).

14. Matter, U.E., Pascual, C., Pretsch, E., Pross, A., Simon, W. and Sternhell, S., TETRAHEDRON , 25,69l (1969).

15. Matter, U.E., Pascual, C., Pretsch, E., Pross, A., Simon, W. and Sternhell, S., TETRAFEDRON , 25,2033 (1969).

16. Uijttewaal, A.P., Jonkers, F.J. and $\overline{\mathrm{Van}}$ del Gen, A., J ORG. CHEM., 44, 3157 (1979).

17. Metzler, $\bar{M}$, TETRAHEDRON, 34,3113 (1978). 